J. Clin. Chem. Clin. Biochem.

Vol. 16, 1978, pp. 283-288

\title{
Kurzprogramme für die Bestimmung von Aminosäuren in physiologischen Flüssigkeiten
}

\author{
Von $K$. Olek, S. Uhlhaas und P. Wardenbach ${ }^{1}$ ) \\ Institut für Humangenetik der Universität Bonn
}

(Eingegangen am 12. Septumber/8. Dezember 1977)

Zusammenfassung: Es werden Kurzprogramme für die Bestimmung von cinigen Aminosäuren gezcigt. Die Arbeiten sind mit zwei im Handel befindlichen lonenaustauscherharzen durchgeführt worden (Durrum DC $G A$, Phoen ix $X X$ 907 OPKU). Alle Programme sind so zusammengestellt, daß sie auch unter unglinstigen Kon\%entrationsverhältınissen Urinanalysen gestatten. Diskutiert werden Wirtschaftlichkeit und Anwendungsmöglichkeiten für solche Verfahren.

\section{Short programs for the determination of amino acids in physiological fluids}

Summary: Short programs for the determination of a few amino acids are described. The studies were performed with two commercially available ion exchange resins (Durrum DC6A, Phoenix XX 907 OPKU). All the programs are constructed so that they are suitable for urine analysis with unfavourable concentration ratios. The cconomic aspects and the areas of application of these methods are discussed.

\section{Einleitung}

Viele klinische Probleme erfordern nicht die quantitative Bestimmung des ganzen Aminosäurenspektrums in physiologischen Flüssigkeiten, sondern nur die Analyse einer oder weniger Aminosäuren. Entsprechende Methoden sollten schnell und wirtschaftlich sein, da in den meisten Fällen große Probenzahlen zu bewältigen sind, wic etwa bei Diätkontrollen oder Screening-Untersuchungen. Man hat deswegen schon vor 10 Jahren Programme für die Ionenaustauscherchromatographie entwickelt, die gerade nur dic gefragten Aminosäuren zu messen gestatten $(1,2)$. Die verwendeten Geräte sind billiger und unkomplizierter als die sogenannten automatischen Aminosäurenanaly. satoren.

Den bisher in der Literatur erschienenen Systemen haften zwei entscheidende Mängel an:

1. Sie sind meistens für lonenaustauscherharze entwickelt worden, die keine große Verbreitung mchr haben oder gar nicht mehr produziert werden $(3,5)$.

2. Die meisten Autoren beschränken sich auf Programme, die offensichtlich nur tauglich sind fur Hydrolysatoder Serumanalysen, nicht aber für den Urin.

\footnotetext{
1) Diese Arbeit wurde unterstutzt vom Bundesministcrium fir Jugend, Familic und Cesundhcit
}

Auch in den Systemen von Shih (1) und Benson $(2,6)$ sind bei kritischen, schwer zu trennenden Substanzen nur dann sichere Messungen möglich, wenn dic interessierenden Aminosäuren in hoher Konzentration vorliegen. Demgegenüber sind die von uns beschriehenen Programme auch bei Normalkonzentrationen für die Urinanalyse anwendbar. Die verwendeten Austauscher. harze sind nach unserer Information inzwischen in sehr vielen Laboratorien in Gebrauch.

\section{Methode}

\section{Verwendete Apparaluren}

Unser Analysator setzte sich aus den folgenden hauptsächlichen Bausteinen zusammen:

Der Programmgeber BT 5052 von der I'irma Biotronik ist normalerweise Teil des von der gleichen Firma vertriebenen Zuck.cranalysators.

2 Dosapro Milton Roy Pumpen Modell I 9633, Ultra-Thermostat $\mathrm{K} 5$ der Firma Colora, Philips-Schrciber PM 8000,

Photometer BT 6620 mit log-lin Wandler, Firma Biotronik, Săulen, Ninhydringefả̧ und Reaktionshad, Firma Biotronik.

Das Reaktionscoil ( $30 \mathrm{~m}$ Länge) und dic Verbindung zum Säulenende besteht aus Teflon-Schlauch mit $0.6 \mathrm{~mm}$ i. D. Zur Entfernung des Ammoniaks wurde cine Vorsüule mit dcm Durrum-Harz DC3 verwendel.

Integrator: Autolab Systcm I 
Ionenaustauscherharze

Durrum DC 6A, Firma Biotronik

Phoenix XX 907 OPKU, Firma Cenco, Haan/Rheinland

Pufferzusammensetzung

Lithiumcitrat kristallin:

Merck 5683

Lithiumchlorid p.a.

Merck 5679

Brij 35

Thiodiglykol

Caprylsäure

Serva 15230

Serva 36170

Merck 193

Lithiumhydroxyd

Merck 5691

11 Puffer enthielt jeweils $2 \mathrm{ml}$ einer aus $35 \mathrm{~g}$ Brij 35 und $100 \mathrm{ml}$ Wasser hergestellten Lösung, $2 \mathrm{ml}$ Thiodiglykol und $0,02 \mathrm{ml}$ Caprylsäure.

\section{Ninhydrinlösung}

Ninhydrin

Zinn-(II)-Chlorid

Merck 6768

Ethylenglykolmonc

Merck 7815

Merck 859

Die Reaktionslösung besteht aus: 31 Ethylenglykolmonomethyläther, 11 Natriumacetat-Puffer $(9441 \mathrm{~g}$ Natriumacetat, $100 \mathrm{ml}$ Eisessig), $80 \mathrm{~g}$ Ninhydrin, $2 \mathrm{~g}$ Zinn-(II)-Chlorid (pulverisiert).

Der Programmgeber gestattete die Vorwahl eines Puffer-Wechsels, der Regenerationsphase und der Äquilibrierphase. Wurde eine große Anzahl von Bestimmungen nötig, so koppelten wir das Gerät mit dem Probenaufgeber und dem Integrator des automatischen großen Aminosäurenanalysators, da es in solch einem Fall natürlich viel wirtschaftlicher ist, eine Probe zur Vollanalyse von Hand einzugeben und die schnellen Analysen vollständig zu automatisieren.

\section{Probenvorbereitung}

Blutplasma wird $1+1$ mit $100 \mathrm{~g} / 1$ Sulfosalicylsäure versetzt, vom Niederschlag wird abzentrifugiert, ein Teil des Überstands wird analysiert.

$5 \mathrm{ml}$ Urin wurden mit $20 \mathrm{mg}$ Sulfosalicylsäure versetzt, der Rückstand wurde abzentrifugiert, ein Teil des Überstandes wurde nach Maßgabe des Kreatiningehaltes auf die Säule gegeben.

\section{Ergebnisse}

\section{System I}

basische Aminosäuren, siehe Abbildung 1 und Tabelle 1.

Die angegebene Packungshöhe scheint optimal zu sein. Säulenlängen um $20 \mathrm{~cm}$ bringen viel höhere Elutionszeiten ( 240 min bis zum Arginin) bei sehr guter Auflösung. Erhöht man den Durchfluß soweit, daß wieder Elutionszeiten von $160 \mathrm{~min}$ für Arginin erreicht werden, kommt man auf einen Säulenvordruck von $100 \mathrm{~atm}$. Ging man bei gleichbleibender Harzmenge auf eine $0,9 \mathrm{~cm}$ Säule über, so erreichte man auch bei Erniedrigung des Durchflußes keine Verbesserung der Elutionszeit, Peakbreite (Empfindlichkeit) und Trennschärfe. Dies steht in einem gewissen Gegensatz zu den Ergebnissen von Mondino (7).

In unseren Händen war das von der Firma Cenco gelieferte Harz etwas weniger tauglich als das DC 6A, was vor allem bei wiederholten Urinanalysen deutlich erkennbar war.
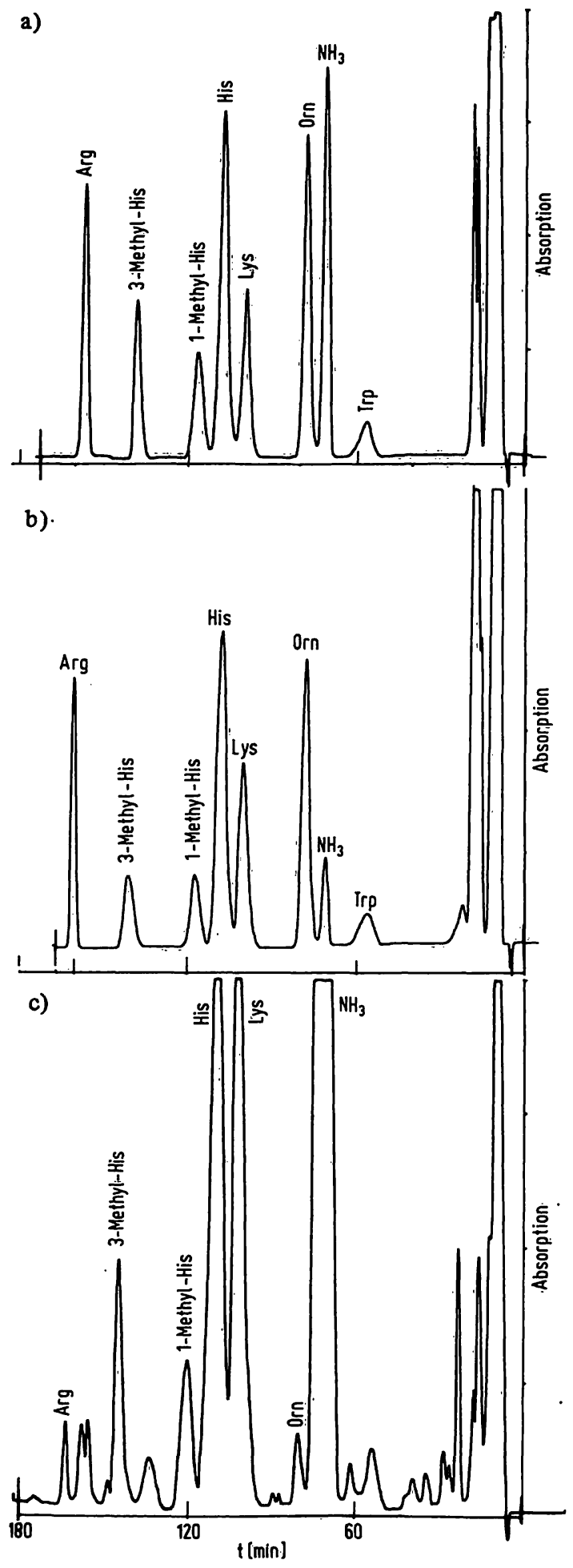

Abb. 1. Aminosäure-Chromatogramme, System I, DC 6A

a) Standardlösung, $200 \mu \mathrm{l}, 250 \mu \mathrm{mol} / 1 \mathrm{je}$ Aminosäure, Absorptionsbereich: 1

b) Normalserum, $500 \mu 1$, Absorptionsbereich: 1

c) Normalurin, $200 \mu \mathrm{l}$, Absorptionsbereich: 1

\section{System II}

Phenylalanin, Tyrosin, Tryptophan, 5-Hydroxytryptophan, 4-Aminobuttersäure, siehe Abbildung 2 und Tabelle 1.

Bei diesem Programm stört das Kreatinin die Bestimmung nicht. Es ist nicht nötig, den Ammoniak aus dem Rohurin zu entfernen. 
Tab. 1. Chromatographiebedingungen und verwendete Harztypen

\begin{tabular}{|c|c|c|c|c|c|}
\hline & System & & & & \\
\hline & I & II & III & IV & $\mathbf{V}$ \\
\hline $\begin{array}{l}\text { Säulentempe- } \\
\text { ratur in }{ }^{\circ} \mathrm{C}\end{array}$ & 40 & 65 & 45 & 46 & 50 \\
\hline $\begin{array}{l}\text { 1. Pufferkon- } \\
\text { zentration in } \\
\text { mol/1 } \\
\text { Lithium- } \\
\text { citrat }\end{array}$ & 0,133 & 0,167 & 0,133 & 0,12 & 0,066 \\
\hline $\begin{array}{l}\text { 1. Puffer-pH- } \\
\text { Wert }\end{array}$ & 4.08 & 4.40 & 3.02 & 2.50 & 2.62 \\
\hline $\begin{array}{l}\text { 2. Puffer-Kon- } \\
\text { zentration in } \\
\text { mol/1 } \\
\text { Lithium- } \\
\text { citrat } \\
\text { Lithium- } \\
\text { chlorid }\end{array}$ & $\begin{array}{l}0,133 \\
0,9\end{array}$ & & & 0,133 & \\
\hline $\begin{array}{l}\text { 2. Puffer-pH- } \\
\text { Wert }\end{array}$ & 4,50 & & & 2,70 & \\
\hline Harztyp & DC6A & $\begin{array}{l}\text { XX } 907 \\
\text { OPKU }\end{array}$ & DC6A & DC6A & DC6A \\
\hline $\begin{array}{l}\text { Harzhöhe } \\
\text { in } \mathrm{cm}\end{array}$ & 16 & 23,2 & 22,0 & 22,0 & 10,5 \\
\hline $\begin{array}{l}\text { Säulendurch- } \\
\text { messer in cm }\end{array}$ & 0,6 & 0,9 & 0,6 & 0,6 & 0,6 \\
\hline $\begin{array}{l}\text { Durchfluß } \\
\mathrm{ml} / \mathrm{h}\end{array}$ & 72 & 110 & 40,8 & 40,8 & 40,8 \\
\hline $\begin{array}{l}\text { Puffer- } \\
\text { wechsel in } \\
\text { Minuten } \\
\text { nach Injek- } \\
\text { tion }\end{array}$ & 115 & - & - & 45 & - \\
\hline $\begin{array}{l}\text { Maximaler } \\
\text { Säulenvor- } \\
\text { druck in } \\
\text { atm }\end{array}$ & 55 & 35 & 75 & 65 & 25 \\
\hline
\end{tabular}

\section{System III}

Norleuciñ (innerer Standard), Leucin, Isoleucin,

Methionin, Cystathioninn, Valin, siehe Abbildung 3 und Tabelle 1.

Bei höherem pH-Ẅẹrt verbessert sich die Trennung zwischen Cystathionin und Methionin, bei niedrigerem pH von Valin und Cystathionin. Die Trennung zwišchèn Isoleucin und Leucin wird bei niedrigerer Temperatur besser.

Das Durrum-Harz żeigte unter allen Bedịngungen in kritischen Fällen etwa 10\% mehr Auflösung.

\section{System IV}

Glycin, Alanin, Citrullin, $\alpha$-Aminobuttersäure, Valin, Cystin, siehe Abbildung 4 und Tabelle s.

J. Clin. Chem. Clin. Biochem. / Vol. 16, 1978 / No. 5

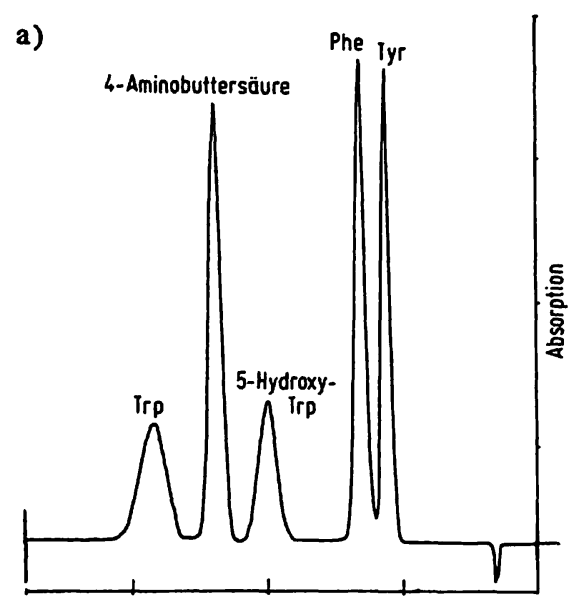

b)

c)
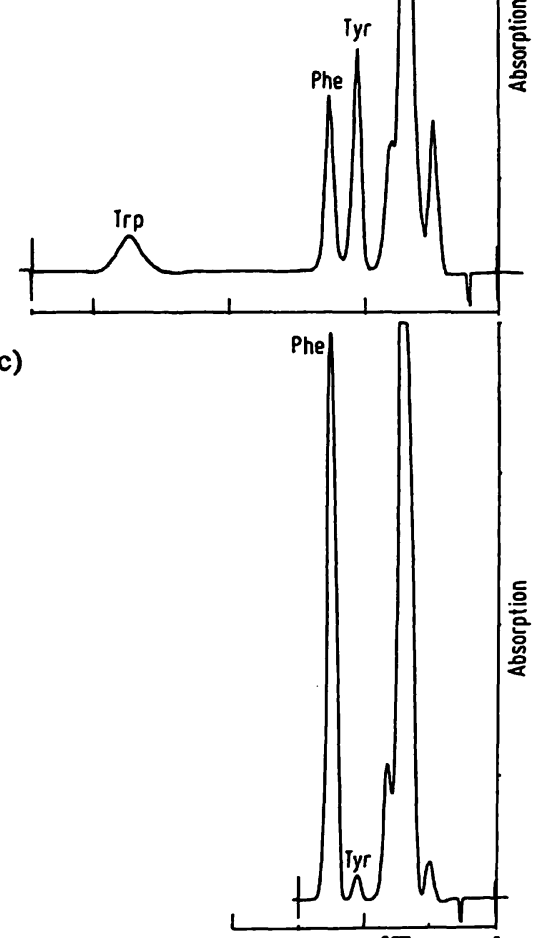

d)

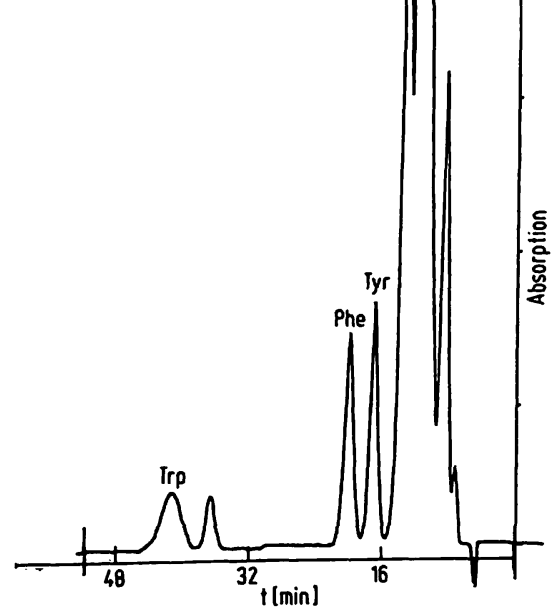

Abb. 2. Aminosäure-Chromatogramme, System II, XX 907-OPKU.

a) Standardlösung, $200 \mu \mathrm{l}, 250 \mu \mathrm{mol} / 1$ je Aminosäure, Absorptionsbereich: 2

b) Normalserum, $500 \mu l$, Absorptionsbereich: 1

c) Phenylketonurie-Serum, $500 \mu \mathrm{l}$, Absorptionsbereich: 2

d) Normalurin, $200 \mu 1$, Absorptionsbereich: 1 


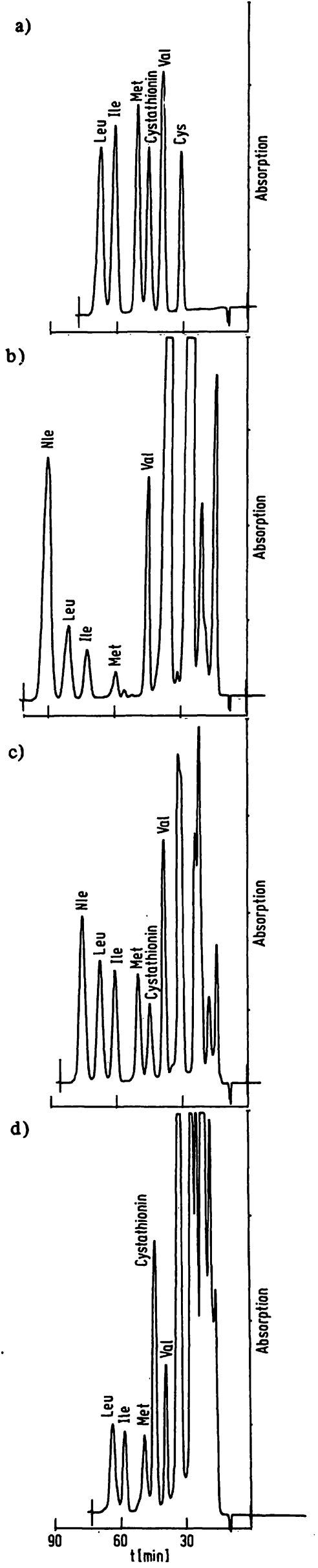

Abb. 3. Aminosäure-Chromatogramme, System III, DĆ 6A.

a) Standardlösung, $200 \mu \mathrm{l}, 250 \mu \mathrm{mol} / 1$ je Aminosäure, Absorptionsbereich: 1

b) Normalserum, $500 \mu$, Absorptionsbereich: 1

c) Normalserum und Cystathionin, $500 \mu 1$, Absorptionsbereich: 1

d) Normalurin, $200 \mu l$, Absorptionsbereich: 1
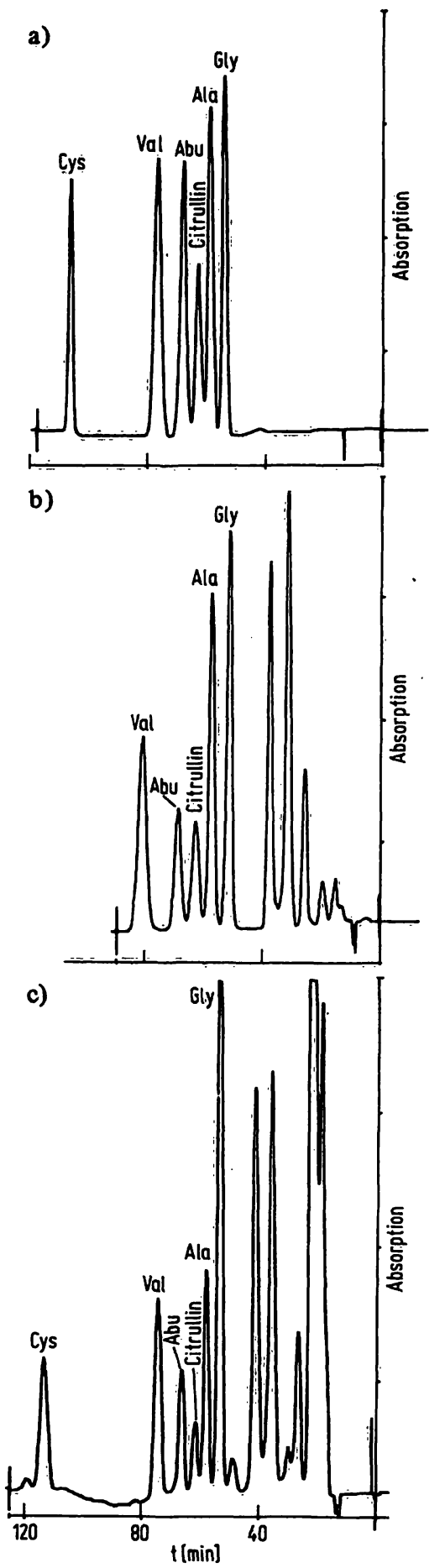

Abb. 4. Aminosäure-Chromatogramme, System IV, DC 6A.

a) Standardlösung $200 \mu 1,250 \mu \mathrm{mol} / 1$ je Aminosäure, 62,5 $\mu \mathrm{mol} / 1$ Citrullin, Absorptionsbereich: 1

b) Normalserum, $500 \mu \mathrm{l}$, Absorptionsbereich: 1

c) Normalurin, $200 \mu \mathrm{l}$, Absorptionsbereich: 1

Mit dem Cenco-Harz war es nicht möglich, Glycin und Alanin vollständig voneinander zu trennen. Bei niedrigerem $\mathrm{pH}=$ Wert werden Alanin und Citrullin besser getrennt, bei höherem Citrullin und $\alpha$-Aminobuttersäure.

\section{System V}

Cystin, Homocitrullin, Methionin, Valin, siehe Abbildung 5 und Tabelle 1.

Auch hier erreichten wir bei kritischen Substanzpaaren etwa $10 \%$ bessere Auflösung mit dem Durnum-Harz.

J. Clin. Chem. Clin. Biochem. / Vol. 16, 1978 / No. 5 

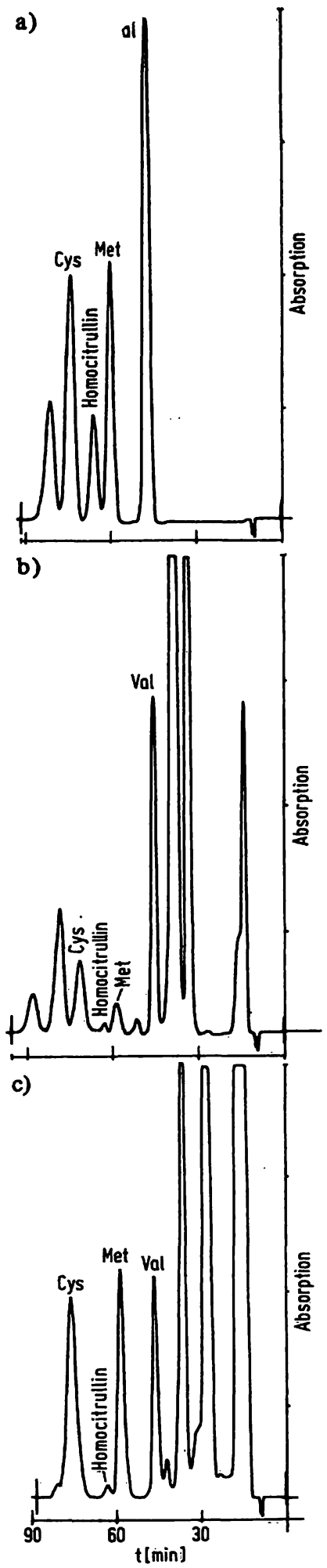

Abb. 5. Aminosäure-Chromatogramme, Systèm V, DC 6A.

a) Standardlösung, $200 \mu \mathrm{l}, 250 \mu \mathrm{mol} / 1$ je Aminosäure, $25 \mu \mathrm{mol} / 1$ Cystin, Absorptionsbereich: 1

b) Normalurin, $500 \mu 1$, Absorptionsbereich: 1

c) Normalurin, $200 \mu \mathrm{l}$, Absor̂́ptioñsbereich: 1
Die Retentionszeitschwankungen für alle dargestellten Gruppen von Aminosäuren liegen beim ungünstigsten Fall, nämlich dem Urin, zwischen 5 und 10\%. Damit ist die automatische Auswertung mit einem elektronischen Integrator immer möglich. Es ist nicht nötig, mit einem internen Standard zu arbeiten. Es ist vielmehr ausreichend, täglich eine Standardlösung zu analysieren. Die Abnahme der Ninhydrinempfindlichkeit beträgt pro Woche $10 \%$. Die Variationskoeffizienten für die einzelnen Bestimmungen liegen zwischen 2 und $10 \%$.

\section{Diskussion}

Nach unseren Informationen sind alle in den letzten Jahren publizierten Analysenprogramme für basische Aminosäuren nicht besser als die von Benson 1972 vorgeschlagenen, wenn man Trennleistung und Dauer der Bestimmung betrachtet. Demgegenüber bedeuten die von uns angegebenen Bedingungen einen Zeitgewinn von rund 40 Minuten bei gleichbleibender Auflösung. Es sind einige Male schnellere Methoden vorgestellt worden, so von Vratny et al. (8) (Arginin etwa 115 Minuten). Urinanalysen sind damit aber ausgeschlossen, weil im Chromatogramm kein Platz für 1-Methylhistidin bleibt.

Ein unserem System II in etwa entsprechendes Programm wird von Mondino (9) und von Berridge (3) et al. gezeigt. Bei ersterem bleibt unklar, wie es sich für physiologische Flüssigkeiten verhält, das zweite dauert bis zur Tryptophanbestimmung 120 Minuten, also 80 Minuten länger als unser System II.

System III bietet die Möglichkeit, die verzweigtkettigen Aminosäuren und Cystathionin auch im Urin zu bestimmen. Dies ist für ein Kurzprogramm nur von Shih (1) beschrieben worden. Dabei wird aber Cystathionin nach 90 Minuten und Leucin nach 110 Minuten eluiert, gegenüber 45 Minuten bzw. 65 Minuten in unserem System.

Ein dem System IV ähnliches Programm findet sich ebenfalls bei Shih et al. (1) mit allerdings wesentlich schlechterer Auflösung zwischen Glycin und Alanin; Citrullin und Glutaminsäure werden dort zusammen eluiert. Unser Programm ist tauglich zur Cystinbestimmung bei der Cystinurie, besser ist allerdings System $V$ verwendbar, in dem auch Homocitrullin gut aufgelöst wird. 


\section{Literatur}

1. Shih, V. E., Efron, M. L. \& Mechanic, G. L. (1967), Anal. Biochem. 20, 299-311.

2. Benson, J. V., Cormick, J. \& Patterson, J. A. (1967), Anal. Biochem. 18, 481-492.

3. Berridge, B. J., jr., Chao, W. R. \& Peters, J. H. (1971), Anal. Biochem. 41, 256-264.
4. Mondino, A. \& Bongiovannini, G. (1971), J. Chromatogr. 63, 411.

5. Jeppson, J. O. \& Karilsșon, J. M. (1972), J. Chromatogr. 72, 93.

6. Benson, J. V. (1972), Anal. Biochem. 50, 477-493.

7. Mondino, A. (1970), J. Chromatogr. $50,260=273$.

8. Vratny, P. \& Zbrozek, J. (1973), J. Chromatogr. 76, 482-486.

Dr. rer. nat. Klaus Olek Institut für Humangenetik der Universität Bonn Wilhelinstraße 31 D-5300 Bonn 1 